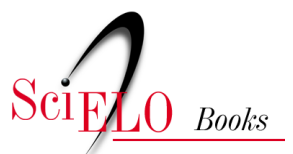

\title{
Vitalidad lingüística del Kichwa Karanki: un estudio desde la documentación activa
}

\author{
Daniela Narváez
}

\section{SciELO Books / SciELO Livros / SciELO Libros}

NARVÁEZ, D. Vitalidad lingüística del Kichwa Karanki: un estudio desde la documentación activa. In: HABOUD BUMACHAR, M., SÁNCHEZ AVENDAÑO, C., and GARCÉS VELÁSQUEZ, F., eds. Desplazamiento lingüístico y revitalización: reflexiones y metodologías emergentes [online]. Quito: Editorial Abya-Yala, 2020, pp. 215-247. Desafíos en la Diversidad collection, n. 2. ISBN: 978-9978-10-541-2. http://doi.org/10.7476/9789978105726.0010.

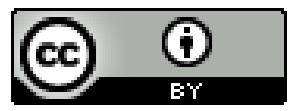

All the contents of this work, except where otherwise noted, is licensed under a Creative Commons Attribution 4.0 International license.

Todo o conteúdo deste trabalho, exceto quando houver ressalva, é publicado sob a licença Creative Commons Atribição 4.0.

Todo el contenido de esta obra, excepto donde se indique lo contrario, está bajo licencia de la licencia Creative Commons Reconocimento 4.0. 


\title{
Vitalidad lingüística del Kichwa Karanki: un estudio desde la documentación activa
}

Daniela Narváez

\begin{abstract}
Resumen
La mayoría de las poblaciones en el mundo se hallan en contacto con más de una lengua. En Ecuador, esta situación de contacto no siempre se ha dado en condiciones de igualdad, lo que ha provocado, desde la conquista española, la imposición del castellano. Como resultado, los pueblos indígenas han sido atacados, invisibilizados y rechazados, lo que a su vez ha ocasionado el desplazamiento de sus lenguas. El kichwa, a pesar de ser la lengua indígena más hablada en el territorio andino, también se ha visto afectado. Uno de los pueblos más importantes de la sierra ecuatoriana que se enfrenta a esta realidad es el pueblo Karanki. Pese a su transcendencia histórica y al hecho de estar en una de las provincias indígenas hablantes de la lengua por excelencia, esta se está perdiendo y casi ha desaparecido entre los jóvenes. Con este trabajo se diagnostica la situación sociolingüística de la lengua kichwa en la provincia de Imbabura, puntualmente en las parroquias Angochagua y la Esperanza. Para esto se ha desarrollado un sondeo sociolingüístico georreferenciado de la vitalidad lingüística del kichwa en esta zona a través de la metodología de la documentación activa. Este estudio consta de 69 entrevistas sociolingüísticas realizadas junto a colaboradores y participantes kichwahablantes indígenas de la misma región. El análisis se desarrolla siguiendo los principales factores para la evaluación de la vitalidad de una lengua propuestos por la UNESCO. Se destacan temas relacionados con el conocimiento y el uso de la lengua en distintos ámbitos comunicativos, el impacto de la escuela en el mantenimiento o pérdida de esta, y varias percepciones de los hablantes.
\end{abstract}

\section{Introducción}

La convivencia y el contacto entre lenguas es un hecho universal que existe desde los tiempos más antiguos. Sin embargo, debido a la globalización, migración y varias situaciones sociopolíticas, económicas y tecnológicas, las fronteras entre pueblos se han vuelto aún más difusas, lo que ha ocasionado que diversas culturas coexistan e

1 Universidad de Massachusetts, Amherst. 
intercambien, no solo sus formas de vida y saberes, sino también sus lenguas. Este contacto no siempre se da en condiciones de igualdad, pues casi siempre las lenguas dominantes se extienden e imponen sobre las de menos prestigio. Esta situación de contacto puede acarrear varias consecuencias, entre ellas, la sustitución, y luego la muerte de una lengua. De hecho, se estima que más de 3200 lenguas en el mundo se encuentran en peligro de extinción (Haboud, 2019; UNESCO 2010; UNICEF, 2009), esto constituye el 50\% de todas las lenguas habladas en la actualidad.

Este escenario de contacto desigual es evidente también en el Ecuador, país intercultural, plurinacional y multilingüe ${ }^{2}$. A pesar de ser uno de los países más pequeños de América del Sur $\left(272045 \mathrm{~km}^{2}\right)$, este cuenta con catorce nacionalidades ${ }^{3}$ y trece lenguas ${ }^{4}$ indígenas (INEC 2010) distribuidas en las tres regiones continentales y en las islas Galápagos (Haboud 2018). Aunque la Constitución del Ecuador (2008) reconoce al castellano, el kichwa ${ }^{5}$ y el shuar como idiomas oficiales de relación intercultural y el Estado se compromete a respetar y a estimular su conservación y uso ${ }^{6}$, sabemos que todas las lenguas del país enfrentan algún grado de desplazamiento hacia el castellano

2 Véase Constitución de la República del Ecuador, 2008.

3 Se considera como nacionalidad indígena a "un conjunto de pueblos milenarios anteriores y constitutivos del Estado ecuatoriano, que se autodefinen como tales, que tienen una identidad histórica, idioma, y culturas comunes, que viven en un territorio determinado mediante sus instituciones y formas tradicionales de organización social económica, jurídica, política y de ejercicio de autoridad" (Chisaguano, 2006, p. 14).

4 De acuerdo con el INEC (2010), las 13 lenguas habladas en el Ecuador son: Achuar, Awapit, A’ingae, Cha'palaa, Siapede, Huao Terero, Secoya, Shuar, Paicoca, Tsa'fiqui, Záparo, Shiwiar y Kichwa.

5 En este estudio se mantiene la ortografía estandarizada de la lengua según consta en la Constitución ecuatoriana: kichwa.

6 Véase Constitución de 2008, Art.2. 
(Haboud, 2007-2018). El castellano7, desde la conquista española en 1532, ha sido, por imposición, la lengua oficial del Ecuador y la tendencia a quitar toda huella de indianidad con el fin de avanzar en el desarrollo de la nación ha sido permanente (Haboud, 2018). Si bien las lenguas indígenas han sido atacadas, invisibilizadas y rechazadas por factores como su estatus de bajo prestigio, falta de exactitud en datos demográficos ${ }^{8}$ y contextos de uso restringido (Calaforra, 2003 en Haboud, 2018), estas continúan vivas en mayor o menor grado, de ahí la importancia de su conservación. El kichwa, puntualmente, a pesar de ser la lengua indígena más hablada a lo largo de los Andes, y que cuenta con más de 10 millones de hablantes (Haboud, 2018), también se ha visto afectado y se lo identifica como minorizado9 debido a su condición de desigualdad en la sociedad (Haboud \& Toapanta, 2014) frente a la hispanización articulada primordialmente por grupos de poder. Uno de los pueblos ${ }^{10}$ kichwas de la sierra que se enfrenta a esta realidad es el pueblo Karanki.

\section{Contextualización}

\section{El pueblo Karanki}

El pueblo Karanki ha sido considerado como una de las culturas más importantes situadas en la sierra ecuatoriana. Su territorio cubría la actual provincia de Imbabura, ascendía hasta la provincia del Carchi e inclusive llegaba hasta el norte de la provincia de Pi-

7 Se usarán los términos "español” y "castellano” como sinónimos.

8 Ver Haboud y King (2007), Mayorga y Haboud (2011), King y Haboud (2012) y Sichra (2009).

9 Ver Calaforra (2003).

10 Existen 13 pueblos dentro de la nacionalidad Kichwa que mantienen su identidad de acuerdo con sus costumbres, dialecto, ubicación geográfica y actividades económicas (SIDENPE, 2008). 
chincha. Sin embargo, tras la conquista incaica se impuso el Kichwa, lo que provocó que su lengua original, el Karanki o Cara, se extinguiera (Costales, 2002). En el siglo XVI, llegaron los conquistadores españoles, quienes, además de imponerles su lengua hegemónica, los despojaron de sus tierras (Shiloh \& Campbell, 2006; Ayala, 2008). Desde esta época, no solo el pueblo Karanki, sino todos los pueblos indígenas del país "han sido víctimas de discriminación por pertenecer a un grupo cultural minoritario y por hablar una lengua que no tiene el mismo prestigio social que el castellano; motivo por el cual se han ido alejando de su cultura y de su lengua con el fin de tratar de ser parte de la sociedad mestiza (Puma, 2017, p.18)”.

En la actualidad, los Karankis están reconocidos como un pueblo dentro de la nacionalidad Kichwa (Costales, 2002; Rhoades, 2006). Según la CONAIE (2014), este pueblo se ubica en la provincia de Imbabura, en las parroquias de San Antonio, Karanki, Angochahua y La Esperanza dentro del cantón Ibarra; en la parroquia San Juan de Ilumán del cantón Otavalo y en las parroquias Mariano Acosta y San Francisco de Sigsipamba del cantón Pimampiro. Sin embargo, esta investigación abarcará únicamente la situación del Pueblo Karanki ubicado en el cantón de Ibarra, parroquias La Esperanza y Angochagua, ya que en estas se encuentra la mayor población que se autorreconoce como Karanki ${ }^{11}$.

Pese a la transcendencia histórica de este pueblo y a pesar de estar localizados en una de las provincias indígenas kichwahablantes por excelencia, como expresa uno de sus líderes jóvenes, "la lengua se ha venido perdiendo desde nuestros padres y casi ha desaparecido entre los jóvenes"12. De ahí que se proponga para este estudio desarrollar un son-

11 Fuente: Censo 2010 y datos recogidos por el Proyecto Oralidad Modernidad en 2013.

12 Elvis Túqueres, líder comunitario en comunicación personal (junio, 2013). 
deo sociolingüístico georreferenciado de la vitalidad de la lengua kichwa entre el Pueblo Karanki en estas dos parroquias de la provincia de Imbabura. Para esto, se analizarán aspectos como la lengua materna de los participantes, las lenguas que conocen y con las que se sienten más cómodos hablando y el uso de estas en diversos ámbitos sociocomunicativos. Además, con estos datos se crearán mapas que ilustren la ubicación de las parroquias y comunidades de estudio y la situación de algunos de los aspectos antes mencionados. Esta documentación constituye el inicio de un proceso de revitalización lingüística del kichwa en esta zona.

\section{Oralidad Modernidad y GeolingüísticaEcuador}

Este trabajo, la metodología que lo subyace y los datos obtenidos pertenecen a los proyectos Oralidad Modernidad ${ }^{13}$ y GeolingüísticaEcuador. Oralidad Modernidad nace en el 2007 con el objetivo de recabar exhaustivamente datos cuantitativos y cualitativos para determinar la situación de la vitalidad de las lenguas indígenas del Ecuador (Haboud, 2007-2019). Parte del principio de desarrollar el trabajo con los propios hablantes para motivar procesos de visibilidad, valorización y revitalización lingüística. Con el fin de determinar la situación de las lenguas de forma más exacta, nace Geolingüística $E$ cuador ${ }^{14}$. En este proyecto se han realizado más de 6700 entrevistas sociolingüísticas georreferenciadas y se han creado más de 1300 mapas que ilustran con bastante exactitud la realidad lingüística que vive el país (Haboud, 2019). A partir de estos datos y resultados nos es posible reflexionar sobre las necesidades específicas de cada lengua, el grado de amenaza en el que se encuentra, y así avanzar con respuestas más precisas en materia social, política y educativa (www.oralidadmodernidad.org).

13 Ver oralidadmodernidad.org/

14 Ver https://oralidadmodernidad.org/geolinguistica/ 


\section{Marco teórico}

La mayoría de las poblaciones del mundo está en contacto con más de una lengua. El escenario de contacto que abarca este estudio, tal y como lo describen Appel y Muysken (1996), es el contacto como resultado de la colonización europea, que ha generado sociedades en las cuales las lenguas europeas de prestigio coexisten con las lenguas indígenas de los pueblos conquistados. Este contacto entre castellano y kichwa es la razón por la cual gran parte de sus hablantes tiende a ser al menos bilingüe (Haboud, 1998, p. 62). Sin embargo, tal y como Haboud (2004, s.p.) describe:

Las lenguas indígenas van siendo desplazadas, y [...] el bilingüismo no es más que una especie de interlengua dirigido al monolingüismo. Las lenguas indígenas se van perdiendo como en goteo permanente (leaking diglosia) en un proceso que se agudiza por su situación de subordinación frente a la sociedad dominante y a la(s) lengua(s) de prestigio.

Es fácil deducir que los que hablan la lengua oficial mayoritaria - el español - y otra lengua de prestigio se reconocen como sujetos bilingües de élite. En cambio, los que además del español hablan una lengua indígena se consideran bilingües minorizados, aún cuando dicha lengua indígena tiene un reconocimiento oficial. En consecuencia, el bilingüismo de élite se reconoce en la sociedad como una posibilidad de avanzar hacia la modernidad y es, por lo tanto, muy valorada, mientras que el bilingüismo minorizado se concibe como un obstáculo para el desarrollo de los pueblos y para la participación en actividades sociopolíticas y socioculturales nacionales, por lo que es blanco de actitudes negativas y destructivas (Haboud 2009: 67-68). El declive de las lenguas indígenas queda demostrado en el hecho de que más y más hablantes de lenguas minorizadas usan la lengua mayoritaria en ámbitos en los que antes habrían usado la vernácula (Appel \& Muysken, 1996, p. 49). 


\section{1}

Debido a esta situación de desigualdad, la vitalidad de las lenguas indígenas del Ecuador se ve afectada. Con el término 'vitalidad' nos referimos al uso real de una lengua por parte de su comunidad de hablantes. Esta es una medida de uso visible y concreta del lenguaje; es decir, cuanto más importantes son las funciones que cumple una lengua para la gran mayoría de los individuos de la comunidad, mayor es su vitalidad (Bouchard et al., 1982, p. 4). Sin embargo, debemos tener en cuenta que la vitalidad se caracteriza por su fuerte fluctuación en el tiempo y el espacio, así como por su difícil determinación y cuantificación (Sichra, 2003, p. 40), de ahí que en este trabajo se tomaron en cuenta los factores para evaluar la vitalidad de una lengua propuestos por UNESCO (2003) en el documento adoptado por la Reunión Internacional de Expertos sobre el programa de la UNESCO "Salvaguardia de las Lenguas en Peligro", titulado "Vitalidad y peligro de desaparición de las lenguas". Si bien estas escalas (ver anexo 1) son útiles para sistematizar los datos, también tienen limitaciones y no pueden ser tomadas como plantillas rígidas. Por lo tanto, para reflejar las diversas problemáticas sociales y su investigación se crearon mapas coropléticos y de puntos en los que se visualizan la ubicación de las lenguas y sus hablantes, su uso, su desplazamiento y las tendencias al mantenimiento o la pérdida, así como, a transmisión intergeneracional, preferencias lingüísticas y uso en varios contextos socio-comunicativos (Haboud, 2018, p. 43) . $^{15}$.

\section{Metodología}

Es importante subrayar la filosofía y principios éticos que subyacen al Programa de Investigación Oralidad Modernidad y, por ende, a esta investigación. El principio con el que se desarrolla este

15 Se puede acceder a todos los mapas relacionados a este trabajo en oralidadmodernidad.org 
programa de investigación interdisciplinaria, como bien lo ha dicho Haboud (2019), es el de la documentación activa en el que los miembros de las comunidades son agentes del proceso de investigación desde sus inicios. Por este motivo, dentro de este estudio se trabajó con entrevistadores indígenas de la zona en cuestión para que se reemplacen los interrogatorios por un diálogo y así los hablantes sean los verdaderos portavoces de su realidad.

En cuanto a la muestra, en un primer momento, se tomaron en consideración los datos del VII Censo de población y VI de Vivienda 2010 en relación con la población autoidentificada como indígena en la provincia de Imbabura ${ }^{16}$. De esta población, se escogió a quienes dijeron hablar la lengua Kichwa. El total de entrevistados por parte del Proyecto fue de 463 personas. Para este trabajo, de esta muestra se escogió la población Kichwahablante de las parroquias de La Esperanza y Angochagua (ver Mapa 1) en donde reside la mayoría de las personas que se autorreconocen como Karankis ${ }^{17}$. El total de participantes fue de 69 personas, 42 de la parroquia La Esperanza ${ }^{18}$ y 27 de Angochagua ${ }^{19}$.

Antes de comenzar el trabajo de campo en la región de estudio, se realizaron encuentros de socialización y capacitación con el equipo participante. En una primera instancia, se revisó la entrevista sociolingüística ${ }^{20}$. Esta fue una oportunidad para afinarla

16 Esta muestra fue elaborada por el Haboud (2009-2010) para la provincia de Imbabura.

17 A pesar de que las instituciones consideran que los Karankis están distribuidos en 8 parroquias; de acuerdo con el censo 2010, la mayor parte de pobladores que se autoidentifica como Karankis se concentra en Angochagua y La Esperanza.

18 Comunidades Cashaloma, Chirihuasi, El Abra, La Florida, Punkuwayko y Rumipamba.

19 Comunidades Chilko, La Merced, Angochagua, La Magdalena, La Rinconada y Zuleta.

20 Entrevista sociolingǘstica diseñada por Büttner y Haboud (1992) y adpatada para las distintas fases de los proyectos desarrollados por Haboud, como Programa Oralidad Modernidad y el proyecto GeolingüísticaEcuador. 
con el equipo de Imbabura gracias a su conocimiento de la zona, sus habitantes y la lengua Kichwa. Este instrumento consta de 95 preguntas, de las cuales 13 son abiertas y 82, cerradas, y se dividen en seis secciones: datos generales, conocimiento y uso de la lengua, cambio lingüístico, variación dialectal, lengua y educación formal y percepciones de la lengua.

\section{Mapa 1}

\section{Distribución de las comunidades y la población entrevistada}

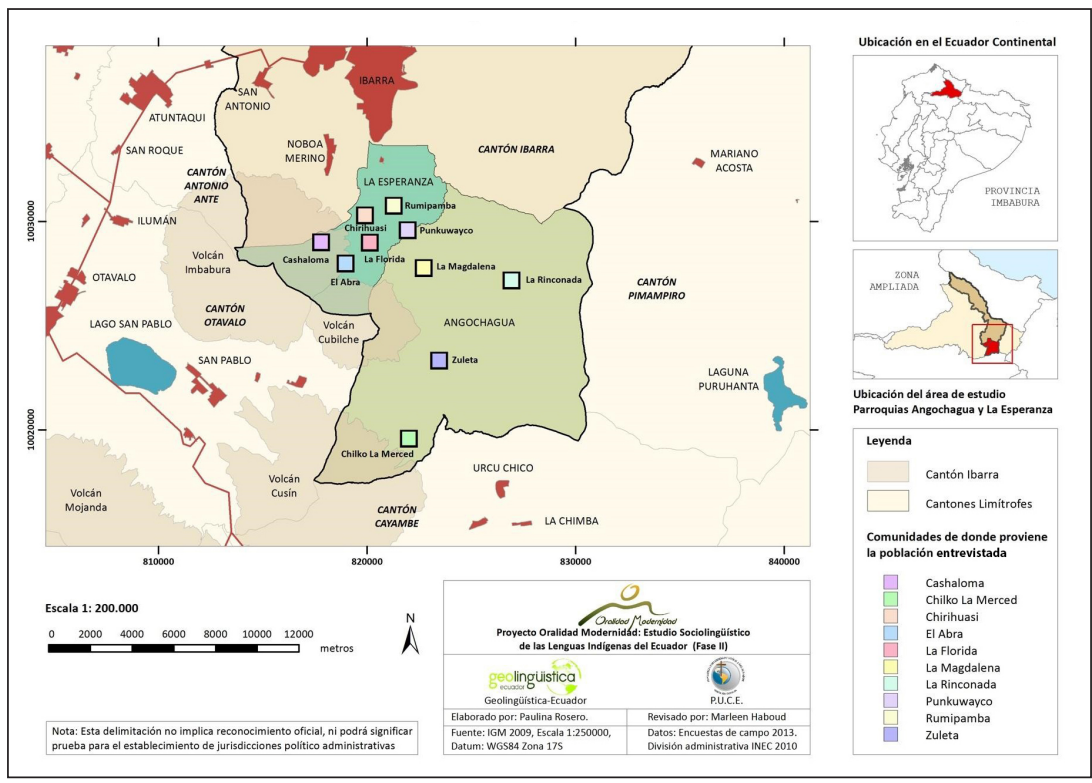

Fuente: Narváez (2014)

Por un lado, la entrevista sociolingüística, así como los datos etnográficos recogidos se organizaron y almacenaron en las plataformas Access y Dropbox. Además, se preparó al equipo investigador en el uso del GPS y así tomar los puntos georreferenciados en cada lugar en el que se realizaron las entrevistas. Una vez que se dio inicio al trabajo 
de campo, las técnicas de investigación empleadas fueron la observación participante, observación no participante, entrevistas sociolingüísticas, guías de observación y conversaciones libres. La observación participante ${ }^{21}$ fue primordial ya que, al formar parte de la vida cotidiana de las comunidades, sin interferir en su normal desenvolvimiento, fue posible palpar el uso auténtico de las lenguas en diversos contextos comunicativos. Así, fue posible llegar a una mejor comprensión de la realidad desde adentro (García \& Giacobbe, 2009, p. 89). Por otro lado, la observación no participante consistió en mirar lo que sucedía en la cotidianidad de las comunidades y registrar los hechos (p. 90 ${ }^{22}$.

Posteriormente, se escogió a los participantes al azar y se realizaron las entrevistas sociolingüísticas ya sea en Kichwa o castellano, según su preferencia. Una vez recabados los datos, para su análisis se tomaron en cuenta los nueve factores que propone UNESCO (2003). $\mathrm{El}$ análisis estadístico de los datos cuantitativos fue realizado con el programa estadístico SPSS. Finalmente, es importante recalcar que el éxito de esta metodología se debe a que, una vez culminado el trabajo, es posible socializar los resultados con las comunidades participantes y, así, crear conciencia sobre la situación en la que se encuentra la lengua Kichwa y, a partir de ello, la misma comunidad pueda desarrollar proyectos que aporten al mantenimiento y revitalización de la lengua.

\section{Análisis de datos y discusión}

A continuación, se analizan los resultados obtenidos a partir de las preguntas y variables ${ }^{23}$ de la entrevista sociolingüística aplicada

21 Para una discusión más profunda en torno a las técnicas de investigación de campo ver García y Giacobbe 2009.

22 Este material se puede encontrar en www.oralidadmodernidad.org

23 Los tres rangos de edad propuestos para este proyecto son mayores de 50, intermedio 30-49 y jóvenes 16-29. 
por el proyecto Oralidad Modernidad en la provincia de Imbabura en el año 2013.

\section{Lengua materna, conocimiento y preferencia}

En el caso de las comunidades de este estudio, el Kichwa es la lengua materna del $71 \%$ de los entrevistados, mientras que el castellano es la primera lengua del $22 \%$ y tan sólo el $7 \%$ asegura tener como lenguas maternas tanto al Kichwa como al castellano. Al relacionar estos datos con los tres rangos de edad, se encontró que existe una correspondencia directa entre la edad y la lengua materna de los entrevistados. Es decir, a mayor edad, la lengua materna es el Kichwa; mientras que, a menor edad, la lengua materna es el castellano (Gráfico 5.1).

\section{Gráfico 5.1 \\ Lengua materna / $\operatorname{edad}^{24}$}

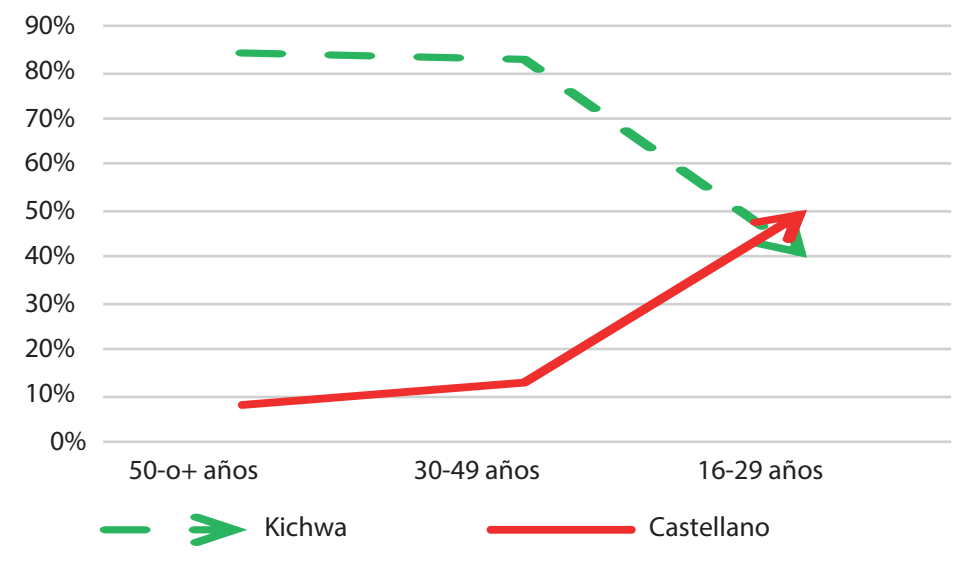

24 A menos de que se indique lo contrario, todas las tablas, cuadros y gráficos de este estudio se basan en el trabajo de campo realizado en las comunidades investigadas por la autora. 
Debido al contacto intenso con el castellano, es compresible que los entrevistados hablen al menos dos lenguas: la minorizada y la de prestigio. El 80\% de los entrevistados asegura hablar Kichwa y castellano; por el contrario, no hay una tendencia alta hacia el monolingüismo o al aprendizaje de otras lenguas. Al correlacionar estos datos con los rangos de edad de los participantes, se obtiene como resultado porcentajes elevados de bilingüismo en todas las edades (Gráfico 5.2).

\section{Gráfico 5.2}

\section{Conocimiento de las lenguas en La Esperanza y Angochagua / edad}

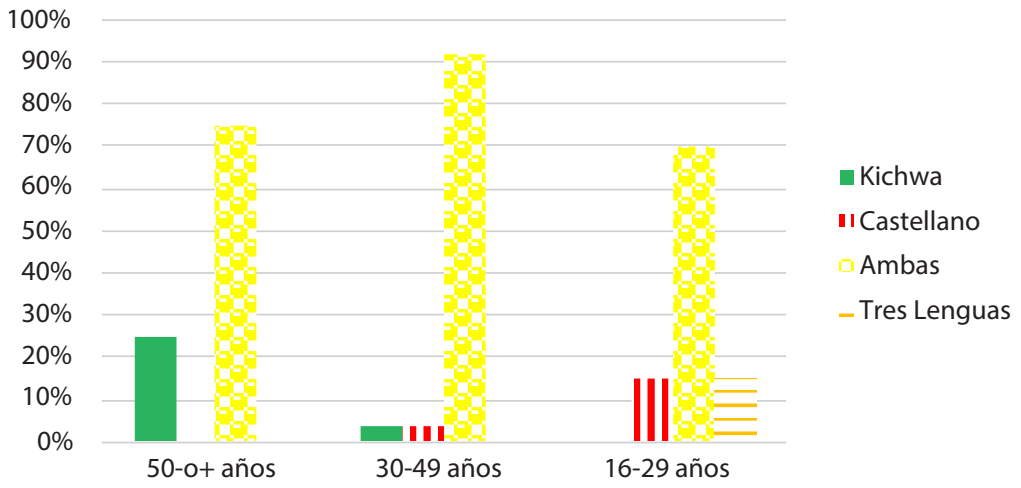

En el rango de 50 o más años se puede apreciar que el 75\% de los hablantes son bilingües; en el rango de 30 a 49 años, el 92\% de los entrevistados son bilingües y en el rango de edad de 16 a 29 años, la cifra de bilingüismo Kichwa-castellano desciende a 70\%, mientras que aumentan los casos de hablantes monolingües de castellano a $15 \%$. La tendencia al bilingüismo se da en las tres generaciones, es decir, la necesidad de los participantes de hablar otra lengua, además del Kichwa, para desenvolverse en los diferentes ámbitos sociocomunicativos no es reciente. Además, se puede advertir una ligera 
inclinación por parte de los más jóvenes por aprender otras lenguas, sobre todo, inglés. Si bien, es una ventaja para los participantes ser bilingües Kichwa-castellano, e inclusive aprender otras lenguas extranjeras, la tendencia hacia la castellanización es cada vez mayor entre el rango de los más jóvenes (16-19 años), comparada con la tendencia de bilingüismo en los otros dos rangos de edad.

Finalmente, al preguntar a los entrevistados su preferencia lingüística, es decir, con cuál de las lenguas se sienten más cómodos hablando, en general, la lengua que prefieren hablar es el Kichwa (54\%), luego está el castellano (26,5\%) y finalmente el 19,1\% de los entrevistados se siente cómodo con las dos. Sin embargo, al observar esta información relacionada con la edad, los resultados son los siguientes (Tabla 5.1):

\section{Tabla 5.1}

Lengua con la que se sienten más cómodos hablando

\begin{tabular}{|l|l|l|l|}
\hline & \multicolumn{1}{|c|}{ Kichwa } & \multicolumn{1}{c|}{ Ambas } & \multicolumn{1}{c|}{ Castellano } \\
\hline 50 o más & $75,0 \%$ & $8,3 \%$ & $16,7 \%$ \\
\hline $30-49$ & $54,2 \%$ & $29,2 \%$ & $16,7 \%$ \\
\hline $16-29$ & $30,0 \%$ & $20,0 \%$ & $50,0 \%$ \\
\hline
\end{tabular}

Son los participantes de mayor edad quienes prefieren hablar kichwa (75\%), mientras que el 50\% de los entrevistados más jóvenes prefieren hablar castellano y tan solo el $20 \%$ se siente más cómodo hablando las dos lenguas. Nuevamente, en estos datos son los más jóvenes quienes se inclinan a hablar solamente el castellano. Estos resultados también se pueden visualizar en el mapa 2 . 


\section{Mapa 2}

Representación de lengua materna, bilingüismo y lengua con la que los entrevistados se sienten más cómodos hablando

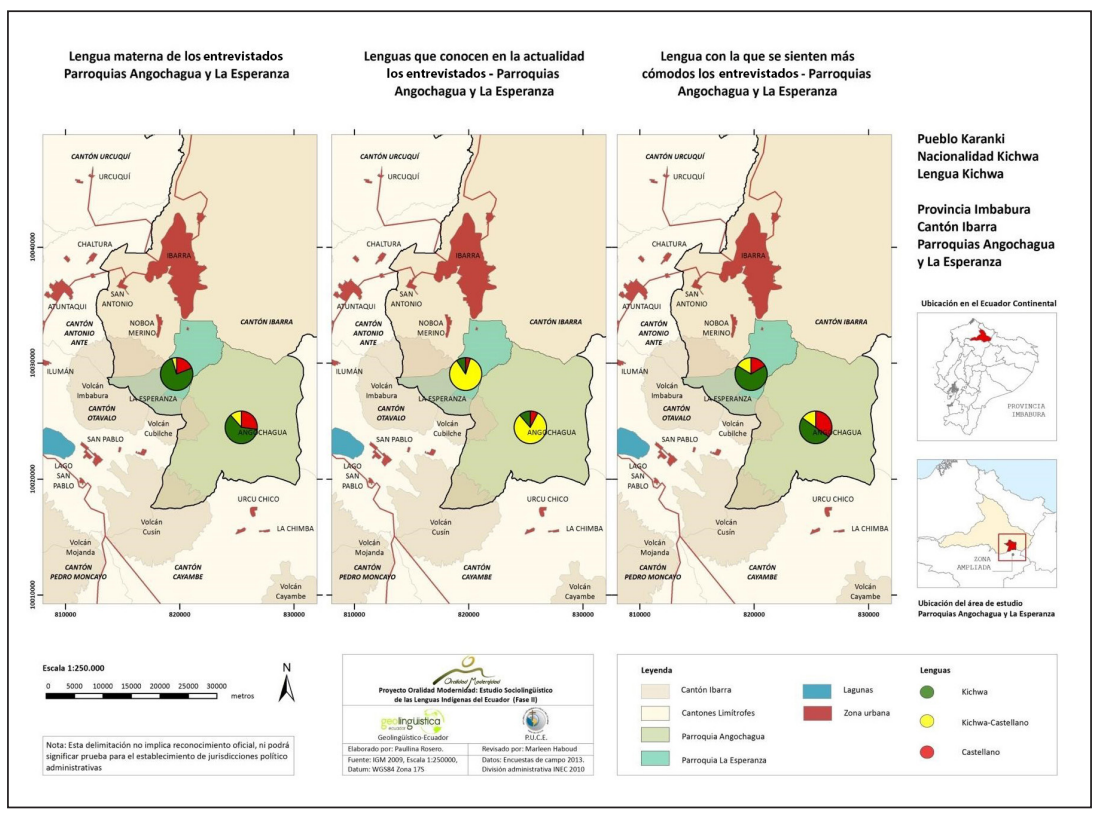

Fuente: Narváez, 2014

Al ver estos resultados, nos preguntamos cuáles son algunas de las causas que inciden en estas preferencias lingüísticas, es decir, cada vez más hablantes se inclinan al monolingüismo (castellano) y no se mantienen como sujetos bilingües (Kichwa-castellano). Entre los factores extralingüísticos que podrían influir en este desplazamiento están la interrupción de la transferencia intergeneracional, el desuso de la lengua en los distintos ámbitos socio-comunicativos dentro y fuera de las comunidades de estudio, el impacto de la educación intercultural bilingüe y la noción que tienen los participantes en cuanto a la realidad lingüística de su comunidad. 


\section{9}

\section{Transmisión intergeneracional}

Siguiendo la metodología planteada por la UNESCO, se continuó el análisis de los datos con el parámetro de transmisión intergeneracional de la lengua dentro del ámbito familiar. Con el fin de conocer el grado de transmisión intergeneracional, el factor central en el mantenimiento del Kichwa, analizamos la lengua que hablan los entrevistados con sus padres y con sus hijos/as. Como bien expone Fishman (1991, pp. 94-95):

La familia es un baluarte imprescindible del proceso de inversión de la sustitución lingüística. La familia tiene un límite natural que sirve de apoyo de las presiones del exterior, costumbres e influencias. Su asociación con la intimidad y lo privado le da una fuerza psicológica y sociológica que la hace especialmente resistente a la competencia y sustitución exterior.

Al cruzar la variable edad con las respuestas que conciernen a la lengua que prefieren hablar en casa, se encuentra que existe una relación directa con las preferencias lingüísticas y el rango de edad. Hallamos que, a mayor edad, hay mayor preferencia por hablar Kichwa y a menor edad, menor preferencia por esta lengua, tal como se detalla en el Gráfico 5.3:

El 75\% de los mayores (50 o más) dice que habla Kichwa en su casa y más de la mitad (54\%) de los del rango intermedio también se comunica en Kichwa en su hogar (54\%). No obstante, entre los más jóvenes (16-29), tan solo el 25\% de los entrevistados dice hablar Kichwa en la unidad doméstica, mientras que el $50 \%$ utiliza solamente el castellano y el 25\% restante las dos lenguas. Estos datos son indicativos de un proceso de sustitución lingüística, ya que en este se observa cómo el uso del castellano aumenta a medida que las generaciones son más jóvenes, mientras que con el Kichwa pasa lo contrario. 


\section{Gráfico 5.3 \\ Lengua que se habla en casa en La Esperanza y Angochagua / edad}

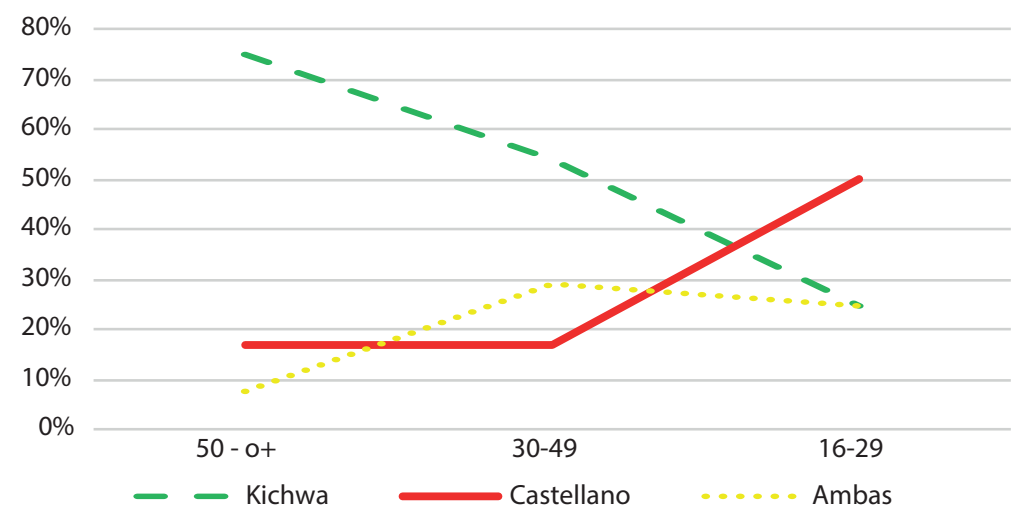

En relación con las lenguas que los entrevistados usan para comunicarse con sus padres, de la totalidad de la población entrevistada, el $71 \%$ dice hablar Kichwa con su padre y su madre, el 19\%, en castellano y el $10 \%$ en ambas. Al momento de cruzar esta información con la edad de los entrevistados, se observa la misma tendencia, a mayor edad, mayor uso del Kichwa y viceversa. Al fijarnos en el rango de edad de los más jóvenes, tan solo el $40 \%$ se comunica en Kichwa con sus padres mientras que el $45 \%$ lo hace en castellano. Por lo tanto, la transmisión intergeneracional ya se empieza a ver afectada en la generación más joven.

Ahora bien, al ver los resultados concernientes a la lengua que usan los participantes para comunicarse con sus hijos según los rangos de edad, notamos, nuevamente, que son negativos para el Kichwa. Incluso en el rango de los mayores (50 o más), en el que en general hemos visto un uso favorable hacia la lengua, cuando se trata de transmitirla a sus descendientes, tan solo el $45 \%$ de los entrevistados dice 
hablar en Kichwa con sus hijos e hijas. Del rango de edad intermedio (30-49), el 26\% de los hablantes dice hablar en Kichwa con sus hijos, el 21,5\% usa el castellano y el 52.5\% las dos lenguas. En el rango de edad de lo más jóvenes (16-29), solamente el 6\% de la población entrevistada habla en Kichwa con sus hijos e hijas, no obstante, el 67\% les habla en castellano y el 27\% en las dos lenguas (Gráfico 5.4).

Gráfico 5.4

\section{Lengua en la que hablan los padres con sus hijos/as}

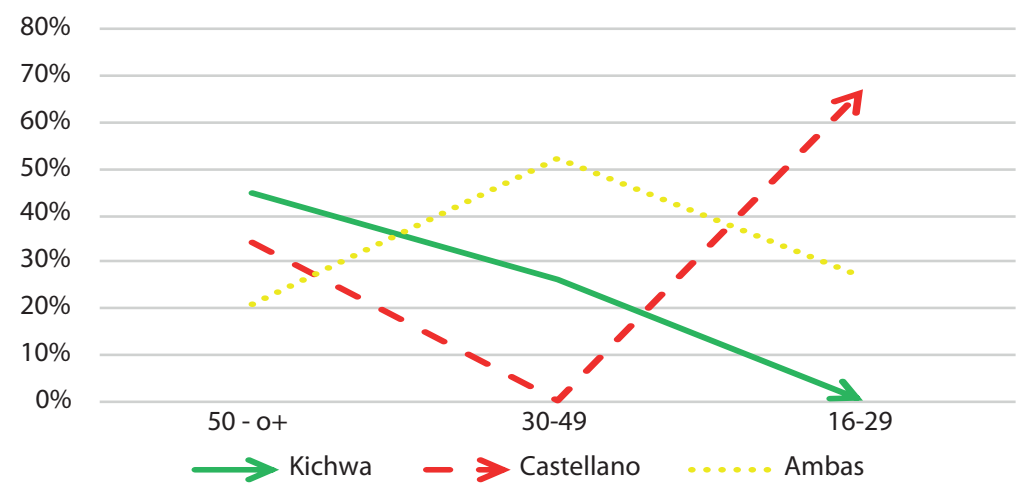

Esto quiere decir que, en la actualidad, la mayoría de los niños y jóvenes de estas dos parroquias no está aprendiendo la lengua vernácula. La lengua es utilizada principalmente por los abuelos y los padres. A partir de lo encontrado con respecto a la transmisión intergeneracional, podemos afirmar que la lengua se ve claramente en peligro o amenazada (3), es decir los niños ya no adquieren el Kichwa como lengua materna y los hablantes más jóvenes pertenecen pues a la generación de los padres. 


\section{Ámbitos sociocomunicativos}

Ámbitos o territorios sociocomunicativos son aquellos contextos en los que se da la interacción sociolingüística. Estos forman una especie de continuum cuyos extremos son el nivel más interno llamado espacio comunitario y el más externo relacionado con un espacio más mestizo (Haboud, 1998, p. 82). Para efectos de este estudio se tomó en cuenta, por un lado, como espacios comunitarios (contextos de fácil acceso para la población indígena) al hogar, ceremonias tradicionales, reuniones comunitarias, amigos kichwas, partidos de fútbol $\mathrm{u}$ otros deportes, autoridades locales y dependiendo de las circunstancias, el lugar de trabajo y estudio. Por otro lado, se consideraron como espacios mestizos (contextos en los que dificulta el uso de la lengua vernácula) la ciudad, el mercado, el hospital y, dependiendo de la situación, el lugar de trabajo y de estudio.

En lo que respecta al ámbito comunitario, si bien se afirma que la lengua minorizada se usa en espacios más privados; es decir, situaciones sociales que por lo general incluyen a grupos de parentesco y sus relaciones más íntimas, la realidad de esta población no se ajusta en su totalidad a esta afirmación. Aunque el Kichwa sigue siendo la lengua de preferencia en la mayoría de los ámbitos comunitarios, la presencia del castellano es más notoria cuando se trata de partidos de fútbol y la comunicación con las autoridades locales (Tabla 5.2).

Tabla 5.2

Uso de las lenguas en la comunidad

\begin{tabular}{|l|l|l|l|}
\hline \multicolumn{1}{|c|}{ Espacios étnicos } & Kichwa & Castellano & Ambas \\
\hline Reuniones Comunitarias & $66 \%$ & $23 \%$ & $11 \%$ \\
\hline Amigos Kichwas & $60 \%$ & $13 \%$ & $27 \%$ \\
\hline Ceremonias tradicionales & $57 \%$ & $31 \%$ & $12 \%$ \\
\hline Partidos de Fútbol & $24 \%$ & $54 \%$ & $22 \%$ \\
\hline Autoridades Locales & $17 \%$ & $55 \%$ & $28 \%$ \\
\hline
\end{tabular}


En relación con las reuniones comunitarias, al cotejar estos datos con la edad, una vez más, el kichwa se ve beneficiado con las generaciones antecesoras (79\%), sin embargo, la mayoría (47\%) de los jóvenes se comunica en castellano. En cuanto a la comunicación cotidiana entre amigos, notamos que los mayores (50 o más) prefieren hablar kichwa $(83,3 \%)$ con sus amistades, así como los del grupo de edad intermedio (50\%). Aunque la mayoría de los jóvenes (40\%) todavía se comunican con sus amigos en kichwa, muchos prefieren hacerlo en castellano (30\%) o en las dos lenguas (30\%), hecho que no era tan común en las generaciones anteriores.

En lo que respecta a las ceremonias tradicionales, advertimos nuevamente que los participantes en el rango mayor y los del rango intermedio usan en su mayoría $(70 \%)$ la lengua vernácula. No obstante, tan solo el 32\% de los más jóvenes habla Kichwa en este ámbito, más de la mitad (58\%) en castellano y el 10\% en ambas (Tabla 5.10). En esta sección cabe preguntarnos ¿qué ceremonias tradicionales predominan en estas parroquias? y ¿cómo influye la religión en la participación de los miembros de las comunidades en fiestas celebradas tradicionalmente en su región? El 65\% de los entrevistados dice ser católico, el 25\% evangélico, tan solo el 1\% Pentecostal y el $9 \%$ no sabe o no tiene una religión. Si bien, a primera vista, parece no haber relación entre el uso de las lenguas y la religión que profesan los participantes, es importante notar que el grupo que se autodenomina católico asiste sobre todo a ceremonias (misa dominical, funerales, bautizos) celebradas en español, fuera de la comunidad. Por otra parte, quienes se adscriben al cristianismo, tienen la opción de evangelizarse en la lengua ancestral, pero, al mismo tiempo no participan en varias de las actividades tradicionales de la comunidad, principalmente en las que se consume alcohol. Sería importante estudiar estos aspectos a mayor profundi- 
dad, tanto en lo que concierne al uso de la lengua, como también al refuerzo cultural.

Por lo que concierne al ámbito deportivo en esta región, sobre todo, partidos de fútbol, tan solo el $24 \%$ de los entrevistados habla en Kichwa cuando juega o alienta al equipo, el $54 \%$ en castellano y el $22 \%$ en ambas. Es preciso mencionar que, de todos modos, varios de los participantes vienen de otras comunidades o de centros urbanos y traen consigo la lengua dominante. Además, como bien sabemos, los deportes en medios de comunicación y en la sociedad en general se transmiten en castellano. En cuanto a la comunicación con autoridades locales, en rasgos generales, únicamente el 17\% de los entrevistados se comunica en Kichwa con estas, el 55\%, en castellano y el $28 \%$ en las dos lenguas. Sin embargo, es importante destacar que varios de los entrevistados plantearon que son las autoridades locales quienes deberían preocuparse por el mantenimiento de la lengua vernácula y que en la actualidad no lo hacen, como se detalla en los siguientes comentarios:

Que las autoridades se preocupen más por el Kichwa, que las personas a quienes siguen el ejemplo, hablen Kichwa, por ejemplo, el presidente de la comunidad, los asambleístas (La Esperanza,-EC 04.13).

Las autoridades deben socializar que el Kichwa es importante y no se pierda (Angochagua, RF 04.13).

Si bien estas opiniones reflejan las actitudes positivas de los hablantes hacia su lengua, en este punto nos cuestionamos si las autoridades de estas comunidades son, en realidad, parte de quienes promueven el mantenimiento de la lengua o, por el contrario, a través de sus prácticas lingüísticas, son quienes promueven el uso del castellano.

Por otro lado, al fijarnos en los resultados correspondientes al ámbito mestizo, los porcentajes más altos del uso del castellano 
corresponden a la ciudad (79\%), al hospital (93\%) y al mercado (81\%); mientras que la lengua nativa casi no se utiliza. Estos espacios propenden a la castellanización aún más acelerada. Al relacionar la información anterior con los rangos de edad, la tendencia general es que el castellano tiene más presencia tanto en el mercado, como en el hospital y en la ciudad. Estos datos muestran la clara hegemonía del castellano en los ámbitos extra-comunales sin que incida en los resultados de la edad de los entrevistados.

En relación con el ámbito laboral, en un sentido general, el 22\% de los hablantes habla Kichwa en su lugar de trabajo, el 53\% en castellano y el 25\% en las dos. Es sencillo asumir que la lengua que se habla en el trabajo depende del lugar en donde esta función se desarrolle. No obstante, los datos demuestran que la realidad es otra y que la tendencia es al uso del español durante las actividades laborales; pues, tanto en la casa como en la ciudad, el porcentaje de uso de la lengua hegemónica es el mismo (55,6\%).

Ya que el español predomina en la mayoría de los espacios tanto étnicos como mestizos, el grado de vitalidad asignado para el caso de los ámbitos socio-comunicativos fue el de ámbitos decrecientes (3) ya que la lengua dominante empieza a penetrar incluso en el ambiente familiar.

\section{Educación intercultural bilingüe en la población Kichwa-Karanki}

Como bien señala Howard (2007), el modelo tradicional de la escuela genera presiones para todas las partes: alumnos, profesores y padres de familia. El utilizar la lengua materna del estudiante, desde los primeros años de educación básica, ha sido ampliamente reconocido por planificadores educativos, y esta es la base de los 
programas de Educación Intercultural Bilingüe (EIB), no solo en el Ecuador, sino en todos los países andinos. Si bien, los objetivos de la EIB plantean tomar en cuenta la diversidad cultural y lingüística de los pueblos y nacionalidades del Ecuador ${ }^{25}$, al observar la realidad en las comunidades de las parroquias La Esperanza y Angochagua, estos objetivos no se cumplen a cabalidad.

En lo que respecta al papel que juega la educación formal en el mantenimiento de la lengua se encontró que, del total de los entrevistados, el 36\% asistió a una escuela bilingüe, el otro 38\% a una escuela hispana, mientras que el 26\% restante no asistió a la escuela. Al correlacionar estos datos con la lengua en la que los entrevistados prefieren hablar fue interesante encontrar que la mayoría de la población escolarizada prefiere hablar castellano. Sin embargo, los que no tuvieron acceso a ningún tipo de educación formal dijeron en su totalidad que prefieren hablar Kichwa. Estos datos reflejan que en estos sectores el objetivo de la EIB de promover el uso de la lengua no se está cumpliendo en su totalidad. Esto no quiere decir que los entrevistados tengan una actitud negativa hacia este tipo de educación en sí. En general, la población de entrevistados, en su mayoría (72\%), opina que el propósito de la EIB está bien o que es positivo; sin embargo, la falla está en su implementación. Como bien mencionan algunos de los entrevistados:

Es bueno pero el nivel de educación actual es deprimente, los profesores no están capacitados (La Esperanza, MS 04.13).

Se supone que deben estar controlados la calidad de educación, pero los niños que salen a los colegios de la escuela bilingüe no son fácilmente aceptados (Angochagua, MA 04.13).

25 Ver educacion.gob.ec/educacion-intercultural-bilingue-princ/ 
Como consecuencia, los niños, las niñas y los jóvenes de las comunidades ya no asisten a escuelas bilingües (39\%) y la mayoría prefiere ir a las escuelas que están ubicadas en las ciudades más cercanas (61\%). En general, estos datos reflejan que los entrevistados no rechazan este tipo de educación, ni el aprendizaje del Kichwa, de modo que habría que aprovechar esta apertura al sistema educativo bilingüe y responder a las expectativas de la población para asegurar el éxito de la EIB y así tener resultados que en realidad aporten con el mantenimiento de las lenguas ancestrales en vez de fomentar su desaparición.

\section{Percepción lingüistica}

También se tomó en cuenta la percepción de los participantes en cuanto a la situación lingüística en su comunidad y las estrategias necesarias para conservar el Kichwa. En general, la percepción que los entrevistados tienen en relación con el uso de la lengua vernácula en la comunidad es que la lengua que más se habla aún es el Kichwa (44\%). El 41\% dice que los habitantes de las comunidades utilizan las dos lenguas y tan solo el $15 \%$ es monolingüe castellano. Ahora bien, al separar la población en tres generaciones: abuelos, padres y jóvenes, la situación se asemeja a los resultados obtenidos en cuanto al uso. Los entrevistados perciben que el 94\% de abuelos y el $85 \%$ de los padres todavía hablan mayoritariamente en Kichwa. Sin embargo, al analizar la lengua que se percibe que los más jóvenes usan, se cree que tan solo el 7\% se comunicaría en Kichwa, mientras que el 74\% lo haría en castellano. Por lo expuesto, podemos plantear que en las parroquias de La Esperanza y Angochagua ya se percibe el desuso de la lengua ancestral, sobre todo en las generaciones jóvenes (Mapa 3). 


\section{Mapa 3}

Percepción lingüística de los entrevistados

del uso de la(s) lengua(s)

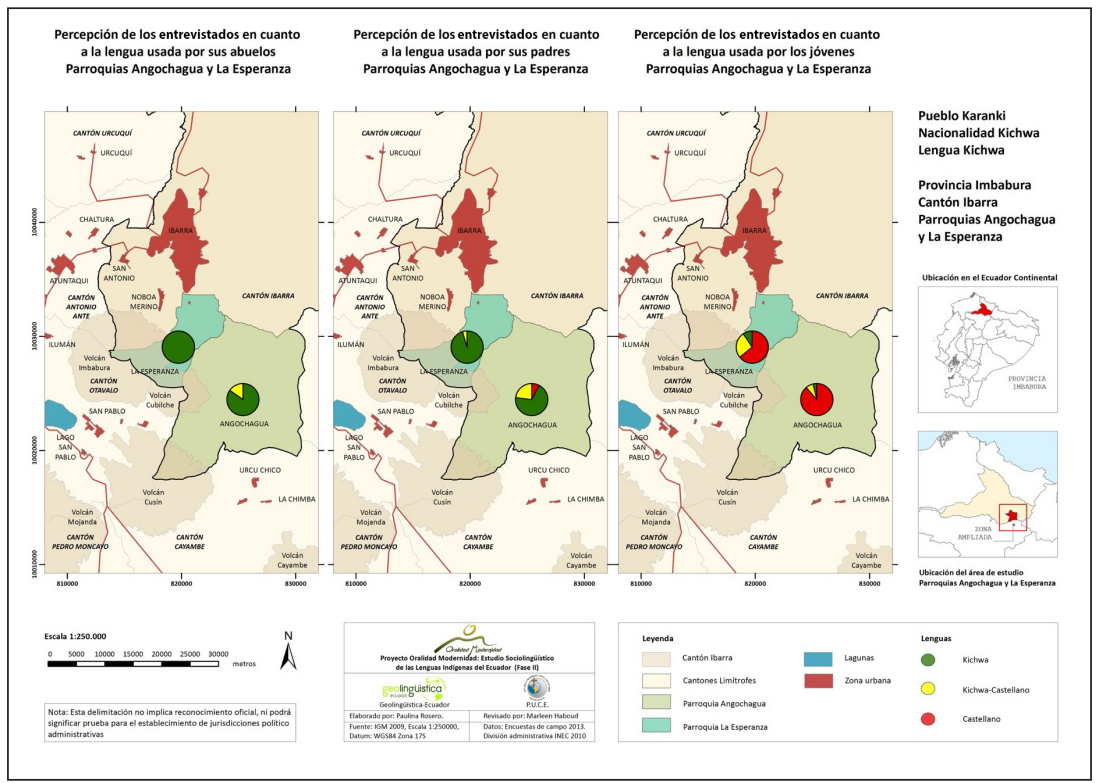

Fuente: Narváez, 2015

\section{Percepción de los entrevistados hacia el futuro del Kichwa}

Ante esta realidad, la mayoría de entrevistados (33\%) opina que una de las acciones que se debe tomar para evitar que la lengua vernácula muera es hablar en Kichwa a los niños y a los jóvenes. Esta forma de pensar coincide con Fishman (1991), quien recalca que el proceso de mantenimiento de una lengua requiere de un cambio social, no solo en los ámbitos educativos o comunitarios, sino más bien es necesario dar prioridad a la transmisión intergeneracional para que haya la posibilidad de detener el desplazamiento: 
Es costumbre hablar en Kichwa, para no perder, las mamás y los papás conversar con los hijos (La Esperanza, MI 04.13).

Enseñar a los hijos desde la casa, hablar en Kichwa los que sabemos (Angochagua, AD 04.13).

Por otra parte, el 26\% recalca la importancia de la Educación Intercultural Bilingüe.

Crear fuentes de trabajo donde se hable Kichwa, escuelas bilingües avanzadas académicamente (Angochagua, MA 04.13).

Enseñar en las escuelas y siempre tome en cuenta esta lengua Kichwa (Angochagua, MC 04.13).

Sin embargo, sabemos que las instituciones educativas bilingües en este sector son castellanizadoras; por lo tanto, es necesario tomar en cuenta la apertura que los participantes tienen hacia la enseñanza de la lengua vernácula y así proponer políticas e iniciativas educativas que realmente sean positivas y efectivas para el mantenimiento de la cultura y la lengua.

Por otro lado, el 20\% de entrevistados afirma que lo que se debe hacer para rescatar la lengua vernácula es simplemente hablarla, hecho que tampoco se da en las actividades diarias:

Deberíamos hablar todos el Kichwa sin miedo (La Esperanza, MT, 04.13).

Toca hablar en Kichwa mismo, a los wawas hacerles aprender, si no hablamos se van perdiendo, a veces ya no entienden, tendríamos nosotros que seguir hablando (Angochagua, MC, 04.2013).

Finalmente, el 23\% restante de entrevistados propone otras opciones para poder mantener su lengua. Una de ellas es que se debe valorar la lengua para conservarla (8\%), otros dicen que es la respon- 
sabilidad de las autoridades (4\%), y algunos proponen la realización de talleres y eventos en Kichwa para promover la lengua y la cultura. $\mathrm{Al}$ preguntar qué pasaría si el Kichwa se pierde, los participantes expresaron las siguientes opiniones:

Se pierde la cultura Kichwa, tanto tiempo luchamos por tener igualdad de derechos como Kichwas y terminamos cediendo lo principal, la identidad ${ }^{26}$, sería algo fatal (La Esperanza, JM 04.13).

También se perdería la vestimenta tradicional, se pierde la identidad cultural no somos ni de allá ni de acá, nos quedamos sin identificación cultural (Angochagua, MA 04.13).

Frente a estas reflexiones, se debe tomar en cuenta la urgencia de dar espacio a las lenguas indígenas y a sus hablantes en contextos que no solo están adscritos a sus comunidades, es decir, en hospitales, mercados, publicidad, señalizaciones oficiales y no oficiales, medios de comunicación, etc. A pesar de que gran parte del esfuerzo para mantener una lengua viene desde las propias comunidades, el hecho de crear visibilidad y conocimiento de la situación de los pueblos y nacionalidades indígenas y sus lenguas, no solo dentro de su territorio, sino en espacios compartidos por indígenas y mestizos, es una responsabilidad tanto del Estado como de toda la sociedad ecuatoriana. Este reconocimiento es primordial para crear una nación realmente intercultural. Como bien señala Ayala Mora (s.f., p.14):

La interculturalidad se construye mediante un esfuerzo expreso y permanente. Va mucho más allá de la coexistencia o el diálogo de culturas; es una relación sostenida entre ellas. Es una búsqueda expresa de superación de prejuicios, racismo, desigualdades, asimetrías que

26 Para un análisis más amplio de la identidad de los datos recopilados para este trabajo, ver Narváez (2014). Para un análisis exhaustivo sobre la relación lengua identidad entre el Kichwa y el pueblo Cañari, ver Enríquez (2015). 
caracterizan a nuestro país, bajo condiciones de respeto, igualdad y desarrollo de espacios comunes.

Por varios años se ha cultivado la idea de que el Ecuador es un país de blancos o blancomestizos, y este imaginario aún sigue bastante arraigado en nuestra sociedad (Ayala Mora, s.f.). Sin embargo, esta visión de la nación ecuatoriana uniforme no es una realidad. El Ecuador es heterogéneo, y por lo tanto esta diversidad cultural, geográfica y lingüística debe ser reconocida, respetada y promovida en todos los ámbitos socio-comunicativos posibles. De no ser así, situaciones de desplazamiento lingüístico, como el caso del Kichwa en este estudio, seguirán ocurriendo. Con estos datos cuanti-cualitativos obtenidos durante el trabajo y a partir de los parámetros de la UNESCO (2003), se constata que la lengua Kichwa entre el pueblo Karanki está en grave peligro.

\section{Conclusiones}

Con este estudio se determinó la vitalidad del Kichwa en el pueblo Karanki de las parroquias La Esperanza y Angochagua mediante un sondeo sociolingüístico georreferenciado. El punto de partida es el contacto lingüístico intenso que tiene el Kichwa de esta zona con el castellano. En relación con los objetivos propuestos para este estudio, y en base a los parámetros que propone la UNESCO, concluimos que la transmisión intergeneracional del Kichwa está muy afectada de modo que los niños ya no aprenden Kichwa como su lengua materna.

El uso de la lengua minorizada en los diferentes ámbitos comunicativos ha cambiado. Es decir, los padres empiezan a utilizar la lengua dominante en su trato cotidiano con los hijos y en el hogar. Así, mientras los padres son bilingües activos que entienden y lo hablan (o saben hablar, aunque no sea su preferencia), los niños pasan a ser bilingües pasivos que entienden, pero no hablan la lengua indígena. 
En cuanto a la educación, en general, las escuelas son entidades castellanizantes y por lo tanto el aprendizaje de la lectura y la escritura en Kichwa no forma parte de los programas escolares. La mayoría de los niños y jóvenes no asisten a las escuelas interculturales bilingües, por lo tanto, no aprenden la lengua vernácula en la escuela, sino castellano y otras lenguas extranjeras de más prestigio. Por estas razones se asignó el Grado 2 de la escala de vitalidad de UNESCO.

En conclusión, la lengua Kichwa entre el pueblo Karanki está en grave peligro, razón por la que el proyecto Oralidad Modernidad junto con varios participantes del pueblo Karanki han continuado su labor en proyectos que fomentan el uso, la valoración y la visibilidad del Kichwa y de sus hablantes. El mantenimiento de una lengua también envuelve conocimientos ancestrales relacionados con la ecología y el medio ambiente. En la actualidad, esto es una preocupación latente, y como bien sabemos las comunidades y pueblos indígenas son, en nuestro país, los principales protectores y transmisores de conocimientos que tienen que ver con el cuidado de la naturaleza como son las formas de cultivo responsable y sostenible, el uso equilibrado de recursos naturales, así como la utilización de plantas medicinales endémicas de cada región del país (Auz, 2018; Sobrevila, 2008). Por lo tanto, Haboud (este volumen) ha estado trabajando en conjunto con varias comunidades de este estudio en la elaboración de léxicos bilingües de plantas medicinales y nativas de esta región.

Al visualizar las reflexiones y los progresos alcanzados en estas comunidades en los últimos años, queda claro que la documentación activa es una metodología que garantiza, si es bien implementa$\mathrm{da}$, que los hablantes sean los protagonistas y voceros de su realidad. Las necesidades de cada comunidad, así como soluciones precisas en torno al mantenimiento y revitalización lingüística y cultural deben ser expresadas desde las mismas localidades. Asimismo, es necesario 
que todos estos esfuerzos en conjunto, avances y experiencias se socialicen, no solo dentro del ambiente académico o tan solo dentro de las comunidades de estudio, sino a nivel nacional y global. De esta manera se puede seguir luchando por que los pueblos indígenas, sus lenguas y sus saberes, que han sido invisibilizados y discriminados por tantos años, obtengan el respeto y el reconocimiento que merecen al ser indispensables para la humanidad.

\section{Referencias bibliográficas}

Almeida, I., Arrobo Rodas, N., \& Ojeda Segovia, L. (2005). Autonomía indígena: frente al Estado Nación y a la globalización neoliberal. Quito: Abya-Yala. Appel, R, \& Muysken, P. (1996). Bilingüismo y contacto de lenguas. Barcelona: Ariel. Auz, J. (2018). Legal empowerment allows indigenous Ecuadoreans to fight multinationals. Open Global Rights.

Ayala Mora, E. (s.f). Interculturalidad en el Ecuador. Quito: Universidad Simón Bolívar. Recuperado de: http://www.uasb.edu.ec/UserFiles/380/File/Interculturalidad\%20en\%20el\%20Ecuador.pdf

Bouchard, E. (1982). Attitudes Towards Language Variation. London: Edward Arnold.

Calaforra, G. Lengua y poder en las situaciones de minorización lingüistica. Recuperado de: http://www.uv.es/calaforr/CursColonia.pdf.

CONAIE (2014). Karankis. Recuperado de: https://conaie.org/2014/07/19/karankis/ Constitución de la República del Ecuador (2008). Recuperado de: http://www. asambleanacional.gov.ec/documentos/constitucion_de_bolsillo.pdf

Chisaguano, S. (2006). La población indígena la población indígena del Ecuador. Análisis de estadísticas socio-demográficas. Quito: Talleres gráficos del INEC.

Costales, A., \& Costales, D. (2002). Huambracuna. La epopeya de Yahuarcocha. Quito: Ediciones Abya-Yala.

Enríquez, P. (2015). El rol de la lengua Kichwa en la construcción de la identidad en la población indígena de Cañar. (Disertación de Licenciatura. Pontificia Universidad Católica del Ecuador. Quito, Ecuador). Recuperado de: http://repositorio.puce.edu.ec/handle/22000/9668

Ferguson, C. (1959). Diglossia. Word, 15, 325-340.

Fishman, J. (1991). Reversing Language Shift: Theoretical and Empirical Foundations of Assistance to Threatened Languages. Multilingual Matters, 76. 


\section{4}

García de Ceretto, J., \& Giacobbe, M. (2009). Nuevos desafios en investigación: Teorías Métodos, técnicas e instrumentos. Ediciones Homo Sapiens.

Giménez, G. (1999). Territorio, cultura e identidades: la región socio-cultural. Colima. Gleich, U. (1989). Educación primaria bilingüe intercultural. Eschborn: GTZ. Haboud, M. (1998). Quichua y Castellano en los Andes Ecuatorianos. Quito: Ediciones Abya-Yala. (2003). ¿Interculturalidad, balance, o sordera visual? En Gugenberger, E. (Ed.), Comunicación intercultural en América Latina: ¿Del conflicto al diálogo? Frankfurt am Main et al.: PeterLang.

(2004). El español y las lenguas indígenas en el Ecuador hoy. En III Congreso Internacional de la Lengua Española. Rosario: Centro Virtual Cervantes.

Proyecto Oralidad Modernidad (Informes 2009, 2010, 2011, 2012). Quito: PUCE (sin publicar) (www.oralidadmodernidad.org) (2014). Vulnerabilidad lingüistica en Ecuador: la lengua awapit. En Zajicová, L. y Zámec, R. (Eds), Lengua y política en América Latina: perspectivas actuales (pp. 119-147). Olomouc: Palacký University, República Checa. (2018). Documentación activa con, desde y para los hablantes de lenguas amenazadas. En Valiente Catter, T., Störl, K. y Gugenberger, E. (Eds.), $L$ a reciprocidad entre lengua y cultura en las sociedades andinas. Estudios de romanistica, lingüística y antropología americana (pp. 37-50). Frankfurt am Main et al.: Peter Lang.

Haboud, M., \& King, K. (2007). Ecuadorian Indigenous Language and Education Policy and Practice: Recent Challenges and Advances. En Bauldauf, R. y Kaplan, R. (Eds.), Language Planning and Policy. Latin America (pp. 105-114). Ecuador, México, Paraguay, Clevedon: Multilingual matters.

Haboud, M., \& de la Vega, E. (2008). El español en América: Ecuador. En Palacios, A. (Coord.), El español en América: Contactos lingüísticos en Hispanoamérica (pp. 161-185). Barcelona: Editorial Ariel S.A.

Haboud, M., \& Ostler, N. (Eds.) (2011). Voices and Images of Endangered. Languages. Bath: Ediciones Lulu.

Haboud, M., \& Toapanta, J. (2014). Voces e imágenes: Lenguas indigenas del Ecuador. Quito: PUCE.

Howard, R. (2007). Por los linderos de la lengua. Perú: Pontificia Universidad Católica del Perú. Fondo Editorial.

INEC (2010). VII censo de población y VI de vivienda 2010. Recuperado de: http:// www.ecuadorencifras.gob.ec/base-de-datos-censo-2010/. 
King, K. A., \& Haboud, M. (2002). Language Planning and Policy in Ecuador. Language Planning and Policy in Latin America, 3(4), 359-424. https:// doi.org/10.1080/14664200208668046

Narváez, D. (2014). Estudio sociolingüistico georeferenciado del kichwa de Imbabura: el caso del pueblo karanki en las parroquias de la Esperanza y Angochahua del cantón Ibarra (Disertación de Licenciatura. Pontificia Universidad Católica del Ecuador. Quito, Ecuador). Recuperado de: http:// repositorio.puce.edu.ec/handle/22000/8236

Puma, C. (2017). Propuesta metodológica para la documentación activa en la comunidad de Chirihuasi. (Disertación de Licenciatura). Quito: Pontificia Universidad Católica del Ecuador. Recuperado de: http://repositorio. puce.edu.ec/bitstream/handle/22000/13858/ (sin publicar)

Rhoades, E. (2006). Desarrollo con identidad: comunidad, cultura, y sustentabilidad en los Andes. Quito: Abya-Yala.

Shiloh, A., \& Campbell, C. (2006). Incursión, fragmentación y Tradición. La ecología histórica de la zona andina de Cotacachi. En Rhoades, R. (Ed.), Desarrollo con identidad. Comunidad, cultura, y sustentabilidad en los Andes (pp. 59-82). Quito: Abya-Yala.

Sichra, I. (2003). La vitalidad del quechua: lengua y sociedad en dos provincias de Cochabamba. Plural editors.

Sobrevila, C. (2008). The Role of Indigenous Peoples in Biodiversity Conservation: The Natural but Often Forgotten Partners. Washington: The World Bank.

UNESCO (2003). Vitalidad y peligro de desaparición de las lenguas. Paris: UNESCO.

(2009). World Atlas of Endangered Languages. Paris: UNESCO.

UNICEF (2009). Atlas Sociolingüistico de pueblos indígenas de América Latina. Cochabamba: FUNPROEIB.

Valiente, T. (1993). Didáctica del quichua como lengua materna. Quito: Abya-Yala. 


\section{Anexos}

Anexo 1

Tabla 8.1

Transmisión intergeneracional de la lengua

\begin{tabular}{|l|c|l|}
\hline Grado de vitalidad & Grado & \multicolumn{1}{|c|}{ Población de hablantes } \\
\hline Vulnerable & 5 & $\begin{array}{l}\text { Todas las generaciones hablan la lengua. No } \\
\text { se ha interrumpido la transmisión de esta en- } \\
\text { tre generaciones. }\end{array}$ \\
\hline $\begin{array}{l}\text { Claramente } \\
\text { en peligro }\end{array}$ & 3 & $\begin{array}{l}\text { La lengua es utilizada por algunos niños en } \\
\text { todos los ámbitos, y por todos los niños en } \\
\text { ámbitos restringidos. }\end{array}$ \\
\hline $\begin{array}{l}\text { Seriamente } \\
\text { en peligro }\end{array}$ & $\begin{array}{l}\text { Los niños ya no aprenden la lengua como } \\
\text { lengua materna. La lengua es utilizada por la } \\
\text { generación parental para arriba. }\end{array}$ \\
\hline En situación crítica & 1 & $\begin{array}{l}\text { Sólo la generación de los abuelos habla la } \\
\text { lengua. La generación de los padres a veces } \\
\text { todavía entiende la lengua pero no la utiliza } \\
\text { para dirigirse a sus hijos. }\end{array}$ \\
\hline Extinta & $\begin{array}{l}\text { Los ancianos sólo recuerdan parte de su len- } \\
\text { gua y no la utilizan de modo permanente, ya } \\
\text { que sólo quedan pocas personas con las que } \\
\text { hablar en ella. }\end{array}$ \\
\hline
\end{tabular}

Fuente: Vitalidad y peligro de desaparición de las lenguas (UNESCO, 2003, p. 7). 
247

\section{Tabla 8.2}

Cambios en los ámbitos de utilización de la lengua

\begin{tabular}{|l|c|l|}
\hline Grado de vitalidad & Grado & \multicolumn{1}{c|}{ Ámbitos y funciones } \\
\hline Uso universal & 5 & $\begin{array}{l}\text { La lengua del grupo etnolingüístico es la lengua } \\
\text { de la interacción, la identidad, el pensamiento, } \\
\text { la creatividad y el entretenimiento. }\end{array}$ \\
\hline $\begin{array}{l}\text { Paridad } \\
\text { plurilingüe }\end{array}$ & 4 & $\begin{array}{l}\text { Una o más lenguas dominantes son las prima- } \\
\text { riamente empleadas en la mayoría de ámbitos } \\
\text { oficiales. Sin embargo, la lengua del grupo et- } \\
\text { nolingüístico puede seguir siendo esencial en } \\
\text { varios ámbitos públicos. }\end{array}$ \\
\hline $\begin{array}{l}\text { Ámbitos } \\
\text { decrecientes }\end{array}$ & 3 & $\begin{array}{l}\text { Los padres empiezan a utilizar la lengua domi- } \\
\text { nante en su trato cotidiano con los hijos en el } \\
\text { hogar, y los niños pasan a ser bilingües pasivos. } \\
\text { Los padres y mayores tienden a ser bilingües ac- } \\
\text { tivos: entienden y hablan las dos. }\end{array}$ \\
\hline Ámbitos limitados & 2 & $\begin{array}{l}\text { Muchos entienden la lengua, pero no la saben } \\
\text { a hablar. }\end{array}$ \\
\hline Ámbitos muy & 1 & $\begin{array}{l}\text { La lengua no dominante se utiliza en ámbitos } \\
\text { muy restringidos por muy pocos miembros de } \\
\text { la comunidad. }\end{array}$ \\
\hline Eximitados & 0 & $\begin{array}{l}\text { La lengua no se utiliza en ningún ámbito para } \\
\text { ninguna función. }\end{array}$ \\
\hline
\end{tabular}

Fuente: Vitalidad y peligro de desaparición de las lenguas (UNESCO, 2003, pp. 8-9) 